\title{
Serum complement 3 is a potential biomarker for assessing disease activity in Takayasu arteritis
}

\author{
Chen Rongyi ${ }^{1,2 \dagger}$, Ma Lingying ${ }^{1,2 \dagger}$, Lv Peng ${ }^{3}$, Lin Jiang ${ }^{3}$, Li Chaolun ${ }^{4}$, Yan Yan ${ }^{1,2}$, Jin Xuejuan ${ }^{5}$, Dai Xiaomin ${ }^{1,2}$,
} Ji Zongfei ${ }^{1,2}$, Chen Huiyong ${ }^{1,2}$, Ma Lili ${ }^{1,2}$, Sun Ying ${ }^{1,2^{*}}$ and Jiang Lindi ${ }^{1,2^{*}}$

\begin{abstract}
Background: Takayasu arteritis (TA) is a rare disease, lacking convenient and feasible biomarkers to identify disease activity. We aimed to evaluate the value of complements in distinguishing active TA.

Methods: Consecutive patients were enrolled from the prospective East China TA cohort from April 2008 to June 2019. Patients were divided into two groups according to their baseline Kerr score. The value of complements and other biomarkers in identifying disease activity were analysed with cluster analysis, ROC curves, and combined tests. An independent group of patients from July 2019 to December 2019 were employed to validate the results.

Results: Of the enrolled 519 patients, 406 (72.2\%) cases were identified as active disease. Higher erythrocyte sedimentation rate (ESR), C-reactive protein (CRP), interleukin-6 (IL-6), and complement 3 (C3) levels were observed in the active group. Elevated C3 ( $\geq 1.085 \mathrm{~g} / \mathrm{L})$ had a high value to identify active TA with a sensitivity of $69.9 \%$, specificity of $66.7 \%$, and AUC of 0.715 . Combining the CRP ( $\geq 10.65 \mathrm{~g} / \mathrm{L}$; sensitivity, $50.7 \%$; specificity, $82.4 \%$ ) and C3, the sensitivity could be improved to $85.1 \%$ in parallel test and the specificity could be improved to $94.1 \%$ in serial test. Validation was further performed to confirm the value of C3 for disease activity assessment. The accuracy of the parallel test of CRP and C3 in external validation with independent 53 TA cases was $72.73 \%$ with the AUC of 0.721 .

Conclusion: Elevated C3 could effectively evaluate the disease activity of TA, and C3 combining with CRP could further improve the disease activity evaluation.
\end{abstract}

Keywords: Complement 3, Takayasu arteritis, Disease activity, Biomarker, C-reactive protein

\section{Background}

Takayasu arteritis (TA), a chronic non-specific inflammatory vascular disease with unknown aetiology, is accompanied by high lethality and morbidity rates [1, 2]. Acute and uncontrolled chronic vascular inflammation would cause vessel wall injury, leading to vascular fibrotic repair in the late phase, and finally resulting in

\footnotetext{
* Correspondence: sun.ying1@zs-hospital.sh.cn; jiang.lindi@zs-hospital.sh.cn

${ }^{\dagger}$ Chen Rongyi and Ma Lingying contributed equally to this work.

'Department of Rheumatology, Zhongshan Hospital, Fudan University, Shanghai, China

Full list of author information is available at the end of the article
}

vascular remodelling $[2,3]$. Therefore, quick and effective treatment is essential to control disease activity and to delay or interrupt vascular inflammation as well as subsequent destruction.

Precise assessment of disease activity plays important roles in the selection and adjustment of treatment strategy. However, valuable biomarkers to evaluate disease activity timely and accurately are still lacking. Traditional activity biomarkers including erythrocyte sedimentation rate (ESR) and C-reactive protein (CRP) are easily affected by other factors such as infections, pregnancy and so on, resulting in poor specificity [4-6].

(c) The Author(s). 2021 Open Access This article is licensed under a Creative Commons Attribution 4.0 International License, which permits use, sharing, adaptation, distribution and reproduction in any medium or format, as long as you give appropriate credit to the original author(s) and the source, provide a link to the Creative Commons licence, and indicate if changes were made. The images or other third party material in this article are included in the article's Creative Commons licence, unless indicated otherwise in a credit line to the material. If material is not included in the article's Creative Commons licence and your intended use is not permitted by statutory regulation or exceeds the permitted use, you will need to obtain permission directly from the copyright holder. To view a copy of this licence, visit http://creativecommons.org/licenses/by/4.0/ The Creative Commons Public Domain Dedication waiver (http://creativecommons.org/publicdomain/zero/1.0/) applies to the data made available in this article, unless otherwise stated in a credit line to the data. 
Previous studies have reported potential biomarkers including matrix metalloproteinases (MMPs) and pentraxin (PTX)3 which were elevated in active TA; however, their values in distinguishing TA disease activity had not been fully illustrated yet, which limited their applications in clinical practice [7]. Thus, it is crucial to find new biomarkers with high sensitivity and specificity to identify disease status of TA.

Complements are important immune molecules in innate immunity and involved in the pathogenesis of vasculitis $[8,9]$. Decreased complement 3 (C3) is a widely used biomarker for active disease in systemic lupus erythematosus (SLE) [10]. Additionally, the downstream cleaved protein of $\mathrm{C} 3$, known as $\mathrm{C} 5 \mathrm{a}$, has become a promising target for ANCA-associated vasculitis (AAV) treatment $[11,12]$. However, the role of complement, especially $\mathrm{C} 3$, in evaluating disease activity of TA has not been investigated yet. So, the aim of this study was to evaluate the potential value of complements in identifying disease activity of Takayasu arteritis.

\section{Methods}

\section{Study design}

A prospectively ongoing observational cohort-the East China Takayasu arteritis (ECTA) cohort-was established since 2010 centred at Zhongshan Hospital, Fudan University, Shanghai, China. All the registered patients in ECTA cohort were diagnosed as TA by experts according to the American College of Rheumatology (ACR) 1990 classification criteria [13]. Patients' information was collected with a standardized form and stored at the Redcap database once diagnosed.

In the current study, baseline information including clinical characteristics, biomarkers, and medications were used for analysis. Patients complicated with the following diseases were excluded: (i) malignant tumour; (ii) acute infections (e.g. tuberculosis, hepatitis); and (iii) other autoimmune diseases (e.g. SLE, AAV). The baseline data of patients enrolled from April 2008 to June 2019 was extracted as the original dataset, while an independent group of patients from July 2019 to Dec 2019 were obtained as the validation dataset. The flowchart of study is listed in the Supplementary Fig. S1.

The investigation protocol was conformed with the Helsinki Declaration and approved by the Ethics Committee of Zhongshan Hospital, Fudan University (Approval No.: B2016-168). All the patients signed the informed consents prior to the enrolment.

\section{Laboratory tests}

Blood samples at baseline of each patient were obtained and examined in the clinical laboratory of the hospital with standard operating procedure. In detail, ESR was detected by Westergren method; CRP as well as globulin and immunoglobulin was detected by automatic biochemical analyser; and cytokines were detected by chemiluminescent immunoassay (CLIA) using Semen platform. Serum complements were detected by immunity transmission turbidity (ITA) in routine procedures according to the instructions of the manufactures with automatic biochemical analyser.

\section{Disease activity evaluation}

Kerr criteria were used as the gold standard for disease activity evaluation: (i) systemic symptoms (infection, tumour, etc., were excluded); (ii) elevated ESR levels; (iii) vascular ischemic symptoms or signs (weakened pulse or pulselessness, vascular bruits, or asymmetric blood pressure); and (iv) positive imaging results. New onset or worsening of two or more criteria indicated "active disease" [14]. Whole body enhanced magnetic resonance angiography (MRA) was performed instead of the traditionally used angiography specified in the Kerr criteria in each patient. Imaging types were identified according to the angiographic classification of the international TA conference in Tokyo (1996) based on lesion distribution [15].

\section{Statistics}

Continuous variables are expressed as the mean \pm standard deviation or median (interquartile range, IQR) and compared using the $t$ test or Wilcoxon rank-sum test where appropriate. Categorical variables are presented as number (frequency) and compared with the chi-square test. The Spearman correlation analysis was employed to validate the relationship between $\mathrm{C} 3$ and other biomarkers. The univariate logistic regression analysis was executed to unearth the factors associated with disease activity, which was further observed reversely by cluster analysis with K-means method and principal component analysis based on the optimal number of groups determined by scree plots. Items with $P<0.05$ in univariate logistic regression analysis were enrolled in the multivariate logistic regression analysis and performed the receiver operating characteristic (ROC) curves. Youden Index was employed to determine the optimal cut-off value and the corresponding diagnosis ability. The parallel test and serial test were further performed to evaluate the value of C3 in combined tests. Next, net reclassification index (NRI) and integrated discrimination index (IDI) were calculated to confirm the value of C3 in improving the ability of CRP or ESR in distinguishing active TA. In addition, the identification effect of the parallel test (CRP and C3) was validated by 10-fold cross-validation and external validation with the original dataset and the independent dataset respectively. A $P<$ 0.05 was considered to be significant with a two-side test. The data were analysed using SPSS 22.0 (Chicago, IL, USA) and R software (Murray Hill, NJ, USA). The graphs were generated with Prism GraphPad 8.0 (San Diego, CA, USA) and R software. 


\section{Results}

\section{Patient characteristics}

In total, 519 patients were enrolled. Of these, 427 cases $(82.3 \%)$ were female. The mean age was 32 (24-45) years, and the median disease course was 21 (4.0-70.5) months. The most common manifestation was dizziness (41.4\%), followed by fatigue (33.3\%) and chest pain (22.2\%). Pulselessness was observed in $41.8 \%$ of patients, vascular bruit in $44.5 \%$ cases, and both were more commonly observed in the active group. The most common imaging type was type $\mathrm{V}$ (35.1\%), followed by type I (28.5\%), type IV (10.2\%), type IIb (7.7\%), type IIa (7.1\%), and type III (3.9\%) (Table 1$)$.

\section{Comparisons of disease characteristics between active and inactive patients}

According to Kerr criteria, 406 cases (72.2\%) were defined as active disease. There was no significant difference between active and inactive groups in age, sex, disease course, and imaging types. Patients with active disease had higher frequency of fatigue, night sweat, chest pain, abdominal pain, pulselessness, and vascular bruit than patients with inactive disease $(P<0.05)$. The prednisone, cyclophosphamide, and methotrexate were the most commonly used drugs in TA patients, but there was no significant difference between two groups (Table 1).

Compared with those in inactive group, complements including C3 [1.00 (0.91-1.15) vs 1.19 (1.03-1.56), $P<$ $0.001]$, C4 $[0.22(0.19-0.27)$ vs $0.25(0.20-0.30), P=$ 0.040 ], and hemolytic complement ( $\mathrm{CH} 50,59.3 \pm 17.9$ vs $66.4 \pm 18.1, P=0.015)$ were significantly higher in the active group. Moreover, serum levels of ESR, CRP, interleukin-6 (IL-6), platelets (PLT), globulin, immunoglobin A (IgA), and immunoglobin G (IgG) were also significantly higher in the active group compared with that in the inactive group $(P<0.05)$ (Table 1$)$.

\section{The relationships between complements and other biomarkers with disease activity}

Then, logistic analysis was performed to clarify the relationships between different biomarkers with disease activity. In univariate regression analysis, it revealed that C3 and CH50, together with PLT, globulin, CRP, IgA, IgG, and IL-6, were positively correlated with disease activity $(\mathrm{OR}>1, P<0.05)$. However, in multivariate regression analysis, C3 levels [odds ratio [OR] (95\%CI) 10.710 (1.825-62.835), $P=0.009$ ] and CRP [OR (95\%CI) 1.041 (1.009-1.073), $P=0.011]$ were independently associated with active disease (Supplementary Table S1).

In the scatter plot, $\mathrm{C} 3, \mathrm{C} 4$, and $\mathrm{CH} 50$ increased with Kerr score significantly and C3 was significantly positively associated with ESR, CRP, and IL-6 $(r>0.4, P<0.001)$ (Fig. 1, Supplementary Fig. S2-3). Furthermore, cluster analysis showed that patients could be clustered into two groups based on
C3, CH50, PLT, globulin, CRP, IgA, IgG, IL-6, ESR, and Hb levels. The principal component analysis revealed that $\mathrm{C} 3$, CH50, CRP, IL-6, PLT, and ESR were assigned to the same major component consisting of inflammatory biomarkers (Fig. 2). These data indicated that C3 levels were associated with the disease activity.

\section{The value of C3 and other biomarkers in identifying disease activity for TA}

In the diagnosis test, the C3 cut-off value for identifying active disease was $1.085 \mathrm{~g} / \mathrm{L}$, with the sensitivity of $69.9 \%$, the specificity of $66.7 \%$, and the AUC of 0.715 (0.650-0.781). In comparison, the cut-off of CRP was $10.65 \mathrm{mg} / \mathrm{L}$, with sensitivity of $50.7 \%$, specificity of $82.4 \%$, and the AUC of 0.703 (0.647-0.760). Moreover, the cut-off of ESR was $26.5 \mathrm{~mm} / \mathrm{H}$, with $63.8 \%$ sensitivity, $73.1 \%$ specificity, and the AUC of 0.766 (0.698$0.800)$. Further combined tests showed that combining CRP and C3 in parallel test, the sensitivity could be improved to $85.1 \%$ and the specificity was $55.0 \%$. In serial test, the specificity was improved to $94.1 \%$, with a sensitivity of $35.4 \%$ (Table 2, Fig. 1).

In addition, results of the NRI [OR (95\%CI) 0.328 (0.224-0.431), $P<0.001$ ] and IDI [OR (95\%CI) 0.389 (0.312-0.466), $P<0.001]$ revealed that the introduction of C3 improved the ability of CRP to distinguish disease activity significantly.

The validation of the value of combination of CRP and C3 in distinguishing active disease

In the internal validation, the 10 -fold cross-validation revealed that the accuracy of the training group was more than $75 \%$ in the parallel test of CRP and C3, among which the maximal accuracy was $90.9 \%$ in the training group with the corresponding accuracy of $83.7 \%$ in the test group (Supplementary Table S2).

In the external validation, 53 cases of independent TA patients were employed to further validate the universality of the results, among whom, 24 (55.8\%) cases were in active status according to the Kerr criteria. There was no significant difference between patients from the original dataset and validation dataset in age, sex, and levels of ESR, CRP, and C3. The AUC of CRP and C3 was 0.721 and 0.692, with the accuracy of $72.0 \%$ and $67.3 \%$, respectively in validation dataset. The AUC was 0.721 with the accuracy of $72.7 \%$ in the parallel test of CRP and C3, while the AUC of serial test of CRP and C3 was 0.721 with the accuracy of $70.4 \%$. The scatter plot also showed that the introduction of $\mathrm{C} 3$ could reclassify some patients with CRP below the cut-off value as active disease (Fig. 3, Supplementary Table S3-4). 
Table 1 The characteristics of TA patients in different disease activity status

\begin{tabular}{|c|c|c|c|c|}
\hline & Total $(n=519)$ & Inactive $(n=113)$ & Active $(n=406)$ & $P$ value \\
\hline Age, years & $32.0(24.0-45.0)$ & $32.0(23.0-44.0)$ & $32.0(24.0-46.0)$ & 0.585 \\
\hline Female, $n(\%)$ & $427(82.3)$ & 89 (78.8) & $338(83.3)$ & 0.269 \\
\hline Course, months & $21.0(4.0-70.5)$ & $20.5(5.0-60.0)$ & $23.0(3.0-71.0)$ & 0.909 \\
\hline \multicolumn{5}{|l|}{ Symptoms } \\
\hline Fever, $n(\%)$ & $71(13.7)$ & $12(11.3)$ & $59(14.8)$ & 0.366 \\
\hline Fatigue, $n(\%)$ & $173(33.3)$ & $29(25.7)$ & $144(35.5)$ & $0.011^{*}$ \\
\hline Night sweat, n (\%) & $16(3.1)$ & $0(0)$ & $16(4.0)$ & $0.030^{*}$ \\
\hline Dizziness, $n(\%)$ & $215(41.4)$ & $44(41.9)$ & $171(43.0)$ & 0.845 \\
\hline Neck pain, $n(\%)$ & $40(7.7)$ & $6(5.6)$ & $34(8.5)$ & 0.316 \\
\hline Chest pain, $n(\%)$ & $115(22.2)$ & $15(14.2)$ & $100(24.9)$ & $0.018^{*}$ \\
\hline Abdominal pain, $n(\%)$ & $14(2.7)$ & $0(0)$ & $14(3.5)$ & $0.048^{*}$ \\
\hline \multicolumn{5}{|l|}{ Signs } \\
\hline Pulseless, n (\%) & $217(41.8)$ & $33(29.2)$ & $184(45.3)$ & $0.034^{*}$ \\
\hline Vascular bruit, $n(\%)$ & $231(44.5)$ & $24(24.0)$ & $207(52.3)$ & $<0.001^{*}$ \\
\hline ESR, $\mathrm{mm} / \mathrm{H}$ & $33.0(14.0-59.0)$ & $15.0(7.0-23.0)$ & $40.0(18.0-66.5)$ & $<0.001^{*}$ \\
\hline Vascular stenosis, $n(\%)$ & $426(82.1)$ & $82(72.6)$ & $344(84.7)$ & 0.882 \\
\hline Vascular thickening, $n(\%)$ & $280(53.9)$ & $40(35.4)$ & $240(59.1)$ & $0.001^{*}$ \\
\hline \multicolumn{5}{|c|}{ Type, $n(\%)$} \\
\hline I & $148(28.5)$ & $32(28.3)$ & $116(28.6)$ & \\
\hline$\| \mathrm{a}$ & $37(7.1)$ & $9(8.0)$ & $28(6.9)$ & \\
\hline$\| \mathrm{b}$ & $40(7.7)$ & $2(1.8)$ & $38(9.4)$ & \\
\hline III & $20(3.9)$ & $5(4.4)$ & $15(3.7)$ & \\
\hline IV & $53(10.2)$ & $15(13.2)$ & $38(9.4)$ & \\
\hline V & $182(35.1)$ & $31(27.4)$ & $151(37.2)$ & 0.063 \\
\hline Hypertension, n (\%) & $111(21.4)$ & $26(23.0)$ & $85(20.9)$ & 0.145 \\
\hline Hyperlipidemia, n (\%) & $13(2.5)$ & $4(3.5)$ & $9(2.2)$ & 0.261 \\
\hline Haemoglobin, g/L & 118.5 (106.0-130.0) & $124.0(115.0-133.0)$ & $117.0(105.0-129.0)$ & $<0.001^{*}$ \\
\hline$W B C, \times 10^{9} / L$ & $7.5(6.0-9.8)$ & $7.3(6.0-8.8)$ & $7.5(6.0-9.9)$ & 0.325 \\
\hline Platelet, $\times 10^{9} / \mathrm{L}$ & $265.0(213.0-342.0)$ & $250.0(210.0-302.5)$ & $275.0(213.5-353.0)$ & $0.048^{*}$ \\
\hline Albumin, g/L & $40.0(37.0-43.0)$ & $42.0(39.0-44.2)$ & $39.0(36.2-42.1)$ & $<0.001^{*}$ \\
\hline Globin, g/L & $29.0(26.0-34.0)$ & $27.0(24.0-33.0)$ & $30.0(26.0-35.0)$ & $<0.001^{*}$ \\
\hline Creatinine, $\mu \mathrm{mol} / \mathrm{L}$ & $58.0(49.0-70.0)$ & $60.0(50.0-73.0)$ & $57.5(49.0-69.0)$ & 0.564 \\
\hline \multicolumn{5}{|l|}{ Immunity-related markers } \\
\hline $\mathrm{CRP}, \mathrm{mg} / \mathrm{L}$ & $7.5(1.9-30.6)$ & $2.4(0.8-8.0)$ & $10.8(2.4-39.1)$ & $<0.001^{*}$ \\
\hline TNF-a, pg/mL & $7.7(5.8-10.2)$ & $7.2(5.9-10.1)$ & $7.9(5.8-10.30$ & 0.692 \\
\hline $\mathrm{IL}-6, \mathrm{pg} / \mathrm{mL}$ & $5.3(2.4-11.2)$ & $3.7(2.1-7.4)$ & $5.7(2.7-12.3)$ & $0.010^{*}$ \\
\hline C3, g/L & $1.15(0.99-1.33)$ & $1.00(0.91-1.15)$ & $1.19(1.03-1.56)$ & $<0.001^{*}$ \\
\hline$C 4, g / L$ & $0.24(0.20-0.29)$ & $0.22(0.19-0.27)$ & $0.25(0.20-0.30)$ & $0.040^{*}$ \\
\hline $\mathrm{CH} 50, \mathrm{~g} / \mathrm{L}$ & $65.2 \pm 18.2$ & $59.3 \pm 17.9$ & $66.4 \pm 18.1$ & $0.015^{*}$ \\
\hline $\operatorname{lgG}, g / L$ & $13.1(10.5-16.3)$ & $11.7(9.4-13.8)$ & $13.3(10.8-6.6)$ & $0.002^{*}$ \\
\hline $\lg A, g / L$ & $2.6(1.8-3.6)$ & $2.3(1.7-3.0)$ & $2.6(1.9-3.8)$ & $0.013^{*}$ \\
\hline $\lg M, g / L$ & $1.4(1.1-2.0)$ & $1.4(1.0-1.8)$ & $1.5(1.1-2.0)$ & 0.160 \\
\hline $\lg E, g / L$ & $26.0(13.0-82.3)$ & $25.0(12.0-70.0)$ & $26.0(13.0-84.5)$ & 0.982 \\
\hline
\end{tabular}


Table 1 The characteristics of TA patients in different disease activity status (Continued)

\begin{tabular}{lllll}
\hline & Total $(\boldsymbol{n}=\mathbf{5 1 9})$ & Inactive $(\boldsymbol{n}=\mathbf{1 1 3})$ & Active $(\boldsymbol{n}=\mathbf{4 0 6})$ & $\boldsymbol{P}$ value \\
\hline Prednisone, $n(\%)$ & $78(15.0)$ & $15(13.3)$ & $63(15.5)$ & 0.502 \\
Methotrexate, $n(\%)$ & $11(2.1)$ & $0(0)$ & $11(2.7)$ & 0.134 \\
Cyclophosphamide, $n(\%)$ & $58(11.2)$ & $17(15.0)$ & $41(10.1)$ & 0.157 \\
\hline
\end{tabular}

ESR erythrocyte sedimentation rate, WBC white blood cells, CRP C-reactive protein, TNF- $a$ tumour necrosis factor-a, IL-6 interleukin-6, C3 complement 3, C4 complement 4, CH50 median hemolytic complement, lgA immunoglobin A, lgG immunoglobin G, $\lg M$ immunoglobin $\mathrm{M}$, $\lg E$ immunoglobin $\mathrm{E}$ ${ }^{*} P<0.05$

\section{Discussion}

To our knowledge, this is the first study to report the value of complement 3 in evaluating disease activity in TA. Our results revealed that elevated C3 had high value in identifying active disease for TA. In addition, the combination of $\mathrm{C} 3$ and traditional biomarker CRP could significantly improve the sensitivity or specificity to distinguish the active TA. One big advantage of the present study was that a validation analysis was performed and further confirmed the value of $\mathrm{C} 3$ for disease activity assessment, though minor differences were observed between original dataset and validation dataset, due to the limited validating sample size.
The convenient and feasible laboratory markers have been explored to evaluate TA disease activity for years, considering the complexity of the imaging examinations [16]. We found that the accuracy of C3 was similar to ESR, but higher than CRP in the present study. Moreover, the sensitivity of $\mathrm{C} 3$ was much better than that of ESR and CRP, which could be validated by an independent group of patients. These data strongly supported that C3 might become a new valuable biomarker for evaluating disease activity in TA.

Inflammation is the foundation of vascular fibrosis and remodelling in TA; thus, assessing disease activity precisely is very important to prevent disease evolution $[2,3]$. We found

\section{A}

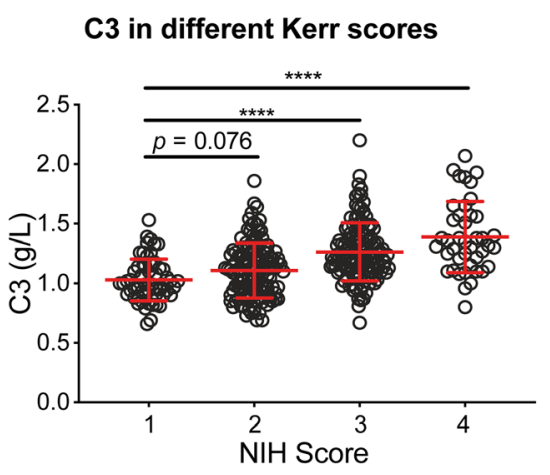

C

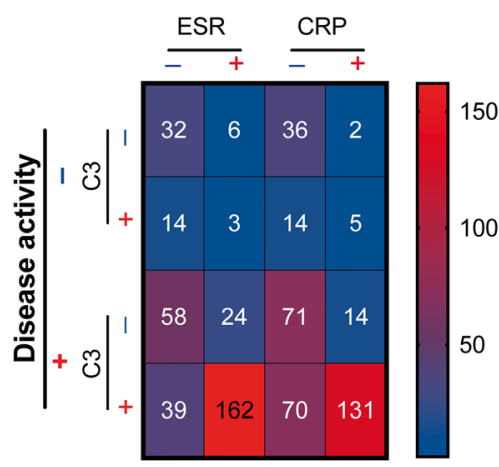

B

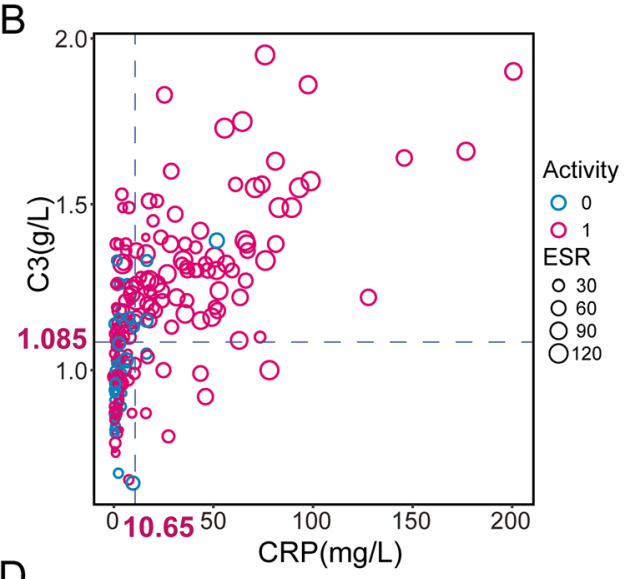

D

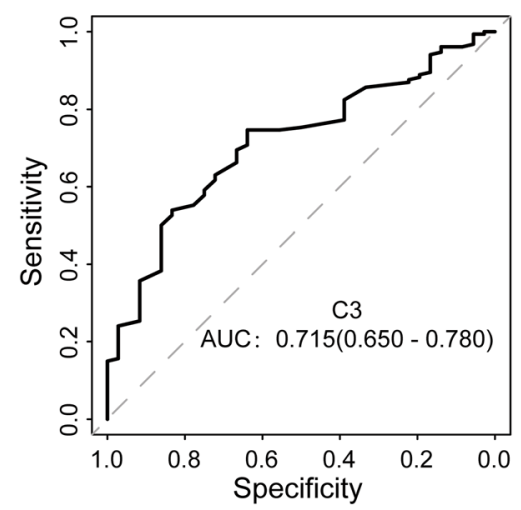

Fig. 1 The value of C3 in identifying TA disease activity. a The distribution of C3 in different levels of Kerr score. b The scatter plot of C3, ESR, CRP, and disease activity (blue line - the cut-off reference line of C3 and CRP; dot size—ESR; dot colour — disease status, "red" indicates active disease status, "green" indicates inactive disease status). c The summary of C3, CRP, ESR, and disease activity in different subgroups based on the cut-off value. $\mathbf{d}$ The ROC curve of C3 to distinguish the disease activity 


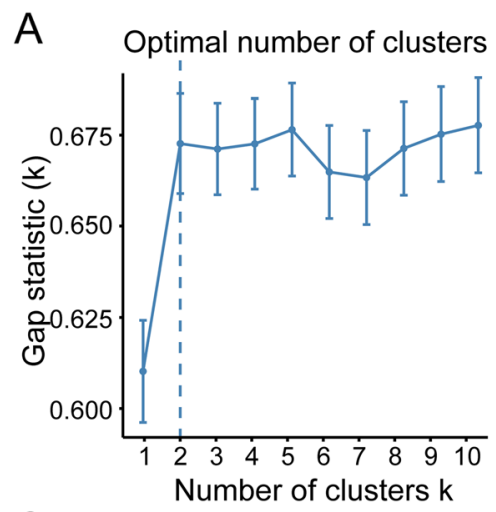

\section{C}

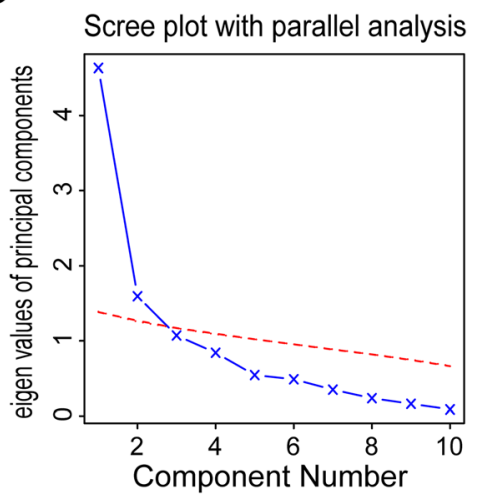

B

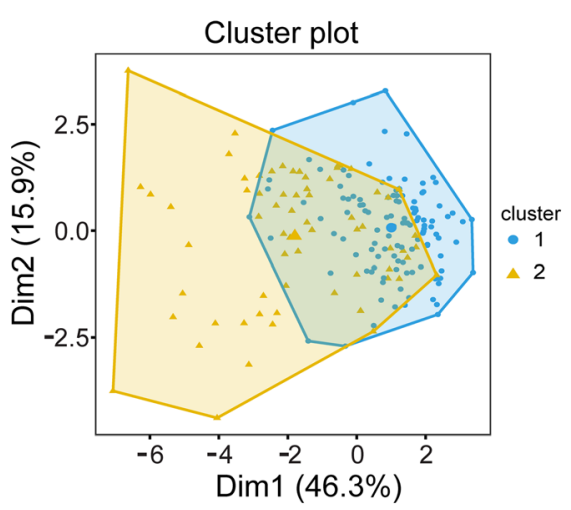

D

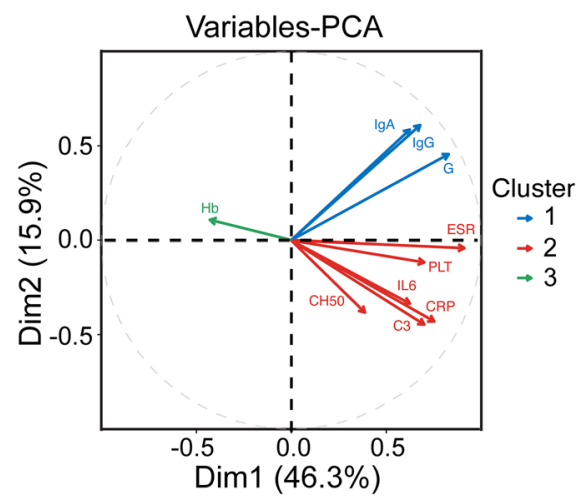

Fig. 2 The cluster analysis and principal component analysis for variables associated with disease activity in TA. a Scree plot to explore the optimal number of the clusters. $\mathbf{b}$ Cluster analysis of the patients. c Scree plot to explore the optimal groups of the principal component analysis. d Principal component analysis based on the variables

that C3 and inflammatory indices including CRP, IL-6, ESR, and PLT belonged to the same component and were positively correlated with active disease and could classify patients into two groups. Moreover, C3 was correlated with CRP, ESR, and IL-6 levels, indicating the role of C3 in evaluating inflammation and identifying disease activity [16]. According to previous reports, IL-6 was involved in the pathogenesis of TA and has become an important intervention target recent years $[17,18]$. However, in our present study, IL-6 showed a relatively lower AUC in identifying active disease, compared with C3.

To improve evaluating disease activity of TA, we performed the parallel and serial tests. The results indicated that the combination of CRP and C3 could significantly improve the sensitivity or specificity to identify active disease in TA. Further validation also confirmed the accuracy of the model in the present study. Considering the multiple components of conventional methods to identify disease activity such as Kerr criteria [19, 20], a single biomarker was difficult to distinguish the active status of the disease for the moment, but combined tests could compensate the shortcomings according to the study. However, which combination was the best strategy was still needed to be further investigated and validated in other cohorts.
C3 might be also involved in the pathogenesis of TA. In TA, the elevated autoantibody anti-epithelial cell antibodies (AECA) serum could mediate the complement-dependent cytotoxicity, leading to the vascular pathogenic lesions [21, 22]. Moreover, infectious antigens might boost the levels of MHC I chainrelated A (MICA), initiating acute inflammation [23]. In this process, proteins such as CRP, targeting microbes, could bind to phosphocholine of the cell membrane, activating C3 through the classical pathway, promoting macrophage elimination of antigens such as debris in active TA [5], resulting in the increased inflammation in the vascular lesions. Accordingly, the upstream protein of $\mathrm{C} 3$ and $\mathrm{C} 4 \mathrm{~b}$ were much higher in the serum of TA as well [16]. These phenomena were also consistent with the finding that $\mathrm{C} 3$ was associated with the active disease. Further mechanisms and causal relations between C3 and other inflammatory index and disease activity should be explored in the future.

There were limitations in the present study. First, the study was based on the ECTA cohort and the results needed to be further validated in other TA cohorts in the future. Second, we found that C3 was valuable to 
Table 2 The sensitivity and specificity of different biomarkers to distinguish the TA disease activity

\begin{tabular}{|c|c|c|c|c|c|c|c|c|}
\hline Marker & AUC & Cut-off value & Sen & Spe & PLR & NLR & PPV & NPV \\
\hline $\mathrm{ESR}^{*}$ & $0.766(0.698-0.800)$ & 26.5 & 0.638 & 0.731 & 2.371 & 0.495 & 0.923 & 0.780 \\
\hline C3 & $0.715(0.650-0.781)$ & 1.085 & 0.699 & 0.667 & 2.099 & 0.451 & 0.911 & 0.667 \\
\hline CRP & $0.703(0.647-0.760)$ & 10.65 & 0.507 & 0.824 & 2.881 & 0.598 & 0.923 & 0.824 \\
\hline Globin & $0.658(0.600-0.716)$ & 29.05 & 0.543 & 0.736 & 2.057 & 0.621 & 0.890 & 0.736 \\
\hline Albumin ${ }^{\#}$ & $0.623(0.556-0.690)$ & 40.25 & 0.667 & 0.581 & 1.592 & 0.573 & 0.721 & 0.345 \\
\hline Haemoglobin ${ }^{\#}$ & $0.620(0.559-0.681)$ & 120.95 & 0.642 & 0.583 & 1.540 & 0.614 & 0.723 & 0.358 \\
\hline $\lg G$ & $0.617(0.549-0.685)$ & 13.32 & 0.503 & 0.736 & 1.905 & 0.675 & 0.895 & 0.736 \\
\hline $\mathrm{CH} 50$ & $0.614(0.529-0.699)$ & 60 & 0.663 & 0.574 & 1.556 & 0.587 & 0.873 & 0.574 \\
\hline IL-6 & $0.610(0.533-0.686)$ & 8.85 & 0.38 & 0.817 & 2.077 & 0.759 & 0.776 & 0.817 \\
\hline $\lg A$ & $0.592(0.524-0.661)$ & 2.865 & 0.438 & 0.743 & 1.704 & 0.756 & 0.881 & 0.743 \\
\hline PLT & $0.566(0.506-0.627)$ & 274.5 & 0.504 & 0.663 & 1.496 & 0.748 & 0.860 & 0.663 \\
\hline \multicolumn{9}{|l|}{ Parallel test } \\
\hline$E S R+C 3$ & $0.683(0.600-0.767)$ & & 0.891 & 0.488 & 1.740 & 0.223 & 0.884 & 0.615 \\
\hline $\mathrm{CRP}+\mathrm{C} 3$ & $0.703(0.623-0.782)$ & & 0.851 & 0.550 & 1.891 & 0.271 & 0.895 & 0.670 \\
\hline $\mathrm{ESR}+\mathrm{CRP}$ & $0.713(0.638-0.788)$ & & 0.822 & 0.602 & 2.066 & 0.296 & 0.901 & 0.710 \\
\hline \multicolumn{9}{|l|}{ Serial test } \\
\hline$E S R+C 3$ & $0.758(0.700-0.816)$ & & 0.446 & 0.910 & 4.956 & 0.609 & 0.982 & 0.964 \\
\hline $\mathrm{CRP}+\mathrm{C} 3$ & $0.703(0.639-0.766)$ & & 0.354 & 0.941 & 6.000 & 0.687 & 0.963 & 0.912 \\
\hline$E S R+C R P$ & 0.699(0.631-0.738) & & 0.323 & 0.953 & 6.832 & 0.710 & 0.960 & 0.918 \\
\hline
\end{tabular}

IgG immunoglobin G, CH50 median hemolytic complement, IgA immunoglobin A, PLT platelet, PLR positive likelihood ratio, NLR negative likelihood ratio, PPV positive predication value, NPV negative prediction value, Sen sensitivity, Spe specificity

${ }^{*}$ ESR belongs to the Kerr criteria and is used as the control here

\#In the ROC curves, the disease activity status discrimination for albumin and haemoglobin is " 0 " (i.e. Inactive disease)

distinguish active disease, compared with $\mathrm{C} 4$ and $\mathrm{CH} 50$, but whether other complement components such as inflammatory $\mathrm{C} 3 \mathrm{a}$ and $\mathrm{C} 5 \mathrm{a}$ could reflect the active disease remained unclear [12].

\section{Conclusions}

In conclusion, $\mathrm{C} 3$ is a potential biomarker for disease activity evaluation in TA, which could also improve the diagnosis ability of other markers.
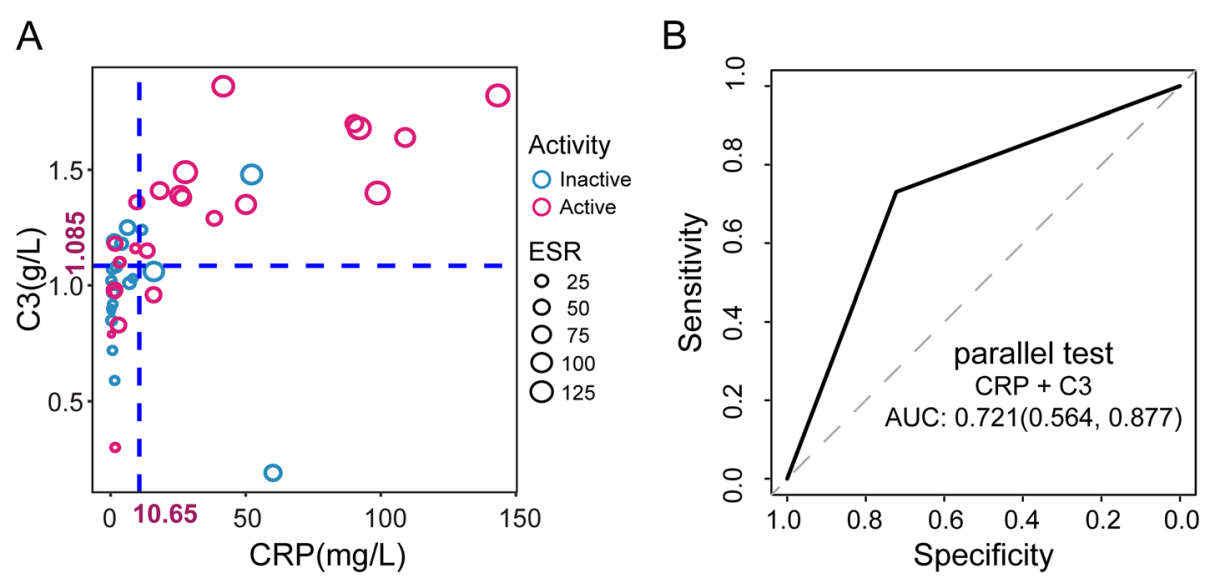

Fig. 3 The external validation of CRP and C3 to identify the active disease based on the validating data in TA. a The distribution of C3, CRP, ESR, and disease activity in validation data (blue line - the cut-off reference line of C3 and CRP in original data; dot colour-disease activity status, "red" indicates active disease status, "green" indicates inactive disease status; dot size-ESR). b The ROC curve of parallel test (CRP and C3) to identify disease activity in validation data of TA 


\section{Supplementary Information}

The online version contains supplementary material available at https://doi. org/10.1186/s13075-021-02433-x.

Additional file 1: Supplementary Table S1. The logistic regression analysis of TA disease activity. Supplementary Table S2. The 10-fold cross-validation to evaluate the accuracy of the parallel test CRP and C3 with the original dataset in the internal validation. Supplementary Table S3. The characteristics of patients in the independent external validation dataset. Supplementary Table S4. The detailed diagnosis result of the external validation. Supplementary Figure S1. The flowchart of the study. Supplementary Figure S2. The distribution of $\mathrm{C} 4$ and $\mathrm{CH} 50$ in different level of Kerr score. Supplementary Figure S3. The correlation analysis of variables.

\section{Abbreviations}

TA: Takayasu arteritis; ESR: Erythrocyte sedimentation rate; CRP: C-reactive protein; C3: Serum complement 3; MMP: Matrix metalloproteinases; PTX3: Pentraxin-3; SLE: Systemic lupus erythematosus; AAV: ANCA-associated vasculitis; NRI: Net reclassification index; IDI: Integrated discrimination index; MRA: Magnetic resonance angiography; ROC: Receiver operating characteristic; IL-6: Interleukin-6; PLT: Platelets; IgA: Immunoglobin A; IgG: Immunoglobin G; OR: Odds ratio; AECA: Anti-epithelial cell antibodies; MICA: MHC I chain-related A

\section{Acknowledgements}

Not applicable.

\section{Authors' contributions}

CRY and MLY contributed equally to the data collection and interpretation and manuscript drafting. LP, LJ, LCL, YY, JXJ, DXM, JZF, and CHY contributed to the clinical work with patients, data collection, and interpretation. MLL and SY contributed to the data interpretation and tables and figures presentation. SY and JLD contributed to the study design and critical revision of the manuscript. JLD sourced funding for this project. The authors read and approved the final manuscript.

\section{Funding}

This work is supported by the National Natural Science Foundation of China [NSFC 81771730 and 81601398].

\section{Availability of data and materials}

The datasets used and/or analysed during the current study are available from the corresponding author on reasonable request.

\section{Ethics approval and consent to participate}

This study was approved by the Ethic Committee of Zhongshan Hospital Fudan University. Written informed consents were obtained when patients were enrolled in the East China Takayasu arteritis cohort (NCT03893136).

\section{Consent for publication}

All participants provided written informed consent prior to their involvement, and the consent for publication has been obtained.

\section{Competing interests}

The authors declare that they have no competing interests.

\section{Author details}

'Department of Rheumatology, Zhongshan Hospital, Fudan University, Shanghai, China. ${ }^{2}$ Evidence-Based Medicine Centre, Fudan University, No.180, Fenglin Road, Xuhui District, Shanghai 200032, China. ${ }^{3}$ Department of Radiology, Zhongshan Hospital, Fudan University, Shanghai, China. ${ }^{4}$ Department of Ultrasound, Zhongshan Hospital, Fudan University, Shanghai, China. ${ }^{5}$ Cardiovascular Epidemiology, Zhongshan Hospital, Fudan University, Shanghai, China.
Received: 9 September 2020 Accepted: 25 January 2021

Published online: 24 February 2021

\section{References}

1. Mirouse A, Biard L, Comarmond C, Lambert M, Mekinian A, Ferfar Y, et al. Overall survival and mortality risk factors in Takayasu's arteritis: a multicenter study of 318 patients. J Autoimmun. 2019;96:35-9.

2. Johnston SL, Lock RJ, Gompels MM. Takayasu arteritis: a review. J Clin Pathol. 2002;55(7):481-6.

3. Vaideeswar P, Deshpande JR. Pathology of Takayasu arteritis: a brief review. Ann Pediatr Cardiol. 2013;6(1):52-8.

4. O'Connor TE, Carpenter HE, Bidari S, Waters MF, Hedna VS. Role of inflammatory markers in Takayasu arteritis disease monitoring. BMC Neurol. 2014;14:1-9.

5. Bray C, Bell LN, Liang H, Haykal R, Kaiksow F, Mazza JJ, et al. Erythrocyte sedimentation rate and $\mathrm{C}$-reactive protein measurements and their relevance in clinical medicine. WMJ. 2016;115(6):317-21.

6. Alende-Castro V, Alonso-Sampedro M, Vazquez-Temprano N, Tunez C, Rey D, Garcia-Iglesias $C$, et al. Factors influencing erythrocyte sedimentation rate in adults: new evidence for an old test. Medicine (Baltimore). 2019;98(34):e16816.

7. Sun Y, Kong X, Wu S, Ma L, Yan Y, Lv P, et al. YKL-40 as a new biomarker of disease activity in Takayasu arteritis. Int J Cardiol. 2019;293:231-7.

8. Vignesh P, Rawat A, Sharma M, Singh S. Complement in autoimmune diseases. Clin Chim Acta. 2017;465:123-30.

9. Charles Jennette J, Xiao H, Hu P. Complement in ANCA-associated vasculitis. Semin Nephrol. 2013;33(6):557-64.

10. Walport MJ. Complement and systemic lupus erythematosus. Arthritis Res. 2002;4(Suppl 3):S279-93.

11. Gou SJ, Yuan J, Chen M, Yu F, Zhao MH. Circulating complement activation in patients with anti-neutrophil cytoplasmic antibody-associated vasculitis. Kidney Int. 2013;83(1):129-37.

12. Peng Q, Li K, Sacks SH, Zhou W. The role of anaphylatoxins C3a and C5a in regulating innate and adaptive immune responses. Inflamm Allergy Drug Targets. 2009;8(3):236-46.

13. Arend WP, Michel BA, Bloch DA, Hunder GG, Calabrese LH, Edworthy SM, et al. The American College of Rheumatology 1990 criteria for the classification of Takayasu arteritis. Arthritis Rheum. 1990;33(8):1129-34.

14. Kerr GS, Hallahan CW, Giordano J, Leavitt RY, Fauci AS, Rottem M, et al. Takayasu arteritis. Ann Intern Med. 1994;120(11):919-29.

15. Hata A, Noda M, Moriwaki R, Numano F. Angiographic findings of Takayasu arteritis: new classification. Int J Cardiol. 1996;54(Suppl):S155-63.

16. Ma J, Luo X, Wu Q, Chen Z, Kou L, Wang H. Circulation levels of acute phase proteins in patients with Takayasu arteritis. J Vasc Surg. 2010;51(3):700-6.

17. Chen R, Sun Y, Cui X, Ji Z, Kong X, Wu S, et al. Autophagy promotes aortic adventitial fibrosis via the IL-6/Jak1 signaling pathway in Takayasu's arteritis. J Autoimmun. 2019;99:39-47.

18. Nakaoka Y, Higuchi K, Arita Y, Otsuki M, Yamamoto K, Hashimoto-Kataoka T, et al. Tocilizumab for the treatment of patients with refractory Takayasu arteritis. Int Heart J. 2013;54(6):405-11.

19. Aydin SZ, Yilmaz N, Akar S, Aksu K, Kamali S, Yucel E, et al. Assessment of disease activity and progression in Takayasu's arteritis with Disease Extent Index-Takayasu. Rheumatology (Oxford). 2010;49(10):1889-93.

20. Direskeneli H, Aydin SZ, Merkel PA. Assessment of disease activity and progression in Takayasu's arteritis. Clin Exp Rheumatol. 2011;29(1 Suppl 64):S86-91.

21. Tripathy NK, Upadhyaya S, Sinha N, Nityanand S. Complement and cell mediated cytotoxicity by antiendothelial cell antibodies in Takayasu's arteritis. J Rheumatol. 2001;28(4):805-8.

22. Mutoh T, Shirai T, Ishii T, Shirota Y, Fujishima F, Takahashi F, et al. Identification of two major autoantigens negatively regulating endothelial activation in Takayasu arteritis. Nat Commun. 2020;11(1):1253.

23. Arnaud L, Haroche J, Mathian A, Gorochov G, Amoura Z. Pathogenesis of Takayasu's arteritis: a 2011 update. Autoimmun Rev. 2011;11(1):61-7.

\section{Publisher's Note}

Springer Nature remains neutral with regard to jurisdictional claims in published maps and institutional affiliations. 